\title{
内蒙古荒漠草原放牧强度对风沙通量和沉积物粒 径的影响
}

李永强 $1,2^{*}$ 李治国 $1^{*}$ 董 智 ${ }^{3}$ 王忠武 ${ }^{1}$ 屈志强 ${ }^{1}$ 韩国栋 $^{1 * *}$

${ }^{1}$ 内蒙古农业大学生态环境学院, 呼和浩特 $010018 ;{ }^{2}$ 土肥资源高效利用国家工程实验室/山东农业大学资源与环境学院, 山东泰安 $271018 ;{ }^{3}$ 山东农业 大学林学院/泰山森林生态站, 山东泰安 271018

摘 要 以内蒙古短花针茅(Stipa breviflora)草原为研究对象, 在放牧11年的样地布设BSNE集沙仪, 通过2013年4月到2015年 4月 4 个采样期对短花针茅草原放牧强度与风沙通量的定量关系及风蚀物粒度特征进行了研究。结果表明: (1)放牧强度对风沙 通量有显著影响 $(p<0.05)$ 。随着放牧强度增强, 风沙通量逐渐增加; 风沙通量对放牧强度的响应存在季节差异, 生长季的日 平均风沙通量小于非生长季, 且生长季不同放牧强度间风沙通量差异较大, 其中重度放牧区风沙通量是对照区的 5 倍, 而非 生长季不同放牧强度间风沙通量差异较小, 重度放牧区是对照区的1.7倍; (2)生长季不同高度风沙通量的自然对数和垂直高 度之间存在较好的二项式拟合结果, 而非生长季风沙通量的垂直分布没有明显规律; 放牧强度间通量的垂直分布差异主要表 现在0-50 cm高度; 在同样高度, 放牧强度大, 垂直通量也大; (3)风蚀沉积物中粒径 $\leqslant 250 \mu \mathrm{m}$ 颗粒富集度随垂直高度变化而变 化; 风沙沉积物中, $\leqslant 250 \mu \mathrm{m}$ 的颗粒占沉积物总量的 $85 \%$ 以上，沉积物中粒径 $\leqslant 50 \mu \mathrm{m}$ 的颗粒有明显的富集作用，且随垂直 高度增加富集比逐渐增加; 粒径为125-250 $\mu \mathrm{m}$ 和50-125 $\mu \mathrm{m}$ 的颗粒表现为随垂直高度增加富集比降低的趋势, 且125-250 $\mu \mathrm{m}$ 粒径的富集比显著小于50-125 $\mu \mathrm{m}$ 颗粒的富集比; 荒漠草原放牧强度对风沙通量有不同程度的影响, 放牧强度越大, 风蚀越 重, 风蚀作用强化了重度放牧对草地退化的影响作用。

关键词 放牧强度; 风蚀; 风沙沉积物; 风沙通量; 荒漠草原

引用格式: 李永强, 李治国, 董智, 王忠武, 屈志强, 韩国栋 (2016). 内蒙古荒漠草原放牧强度对风沙通量和沉积物粒径的影响. 植物生态学报, 40, 1003-1014. doi: 10.17521/cjpe.2016.0045

\section{Effects of grazing intensity on windblown sediment mass flux and particle size distribution in the desert steppe of Nei Mongol, China}

LI Yong-Qiang ${ }^{1,2 *}$, LI Zhi-Guo ${ }^{1 *}$, DONG Zhi ${ }^{3}$, WANG Zhong-Wu ${ }^{1}$, QU Zhi-Qiang ${ }^{1}$, and HAN Guo-Dong ${ }^{1 * *}$

${ }^{1}$ College of Ecological and Environmental Sciences, Inner Mongolia Agricultural University, Hohhot 010018, China; ${ }^{2}$ National Engineering Laboratory for Efficient Utilization of Soil and Fertilizer Resources; College of Resources and Environment, Shandong Agricultural University, Taian, Shandong 271018, China; and ${ }^{3}$ Forestry College of Shandong Agriculture University, Taishan Forestry Ecological Station, Taian, Shandong 271018, China

\section{Abstract}

Aims Grazing activities degrade soil aggregates, reduce vegetation coverage and affect the amount of deposited material, and make the land more vulnerable to wind erosion. Although livestock increase was considered as the main issue leading to the degradation, only very few studies have quantitatively investigated the relationship between grazing and soil erosion. The relationship between different stocking rates and sediment flux, and sediment soil particle was studied to reveal the mechanism of different grazing intensities on soil erosion process, to provide basic parameters for grazing optimization in the Stipa breviflora desert steppe.

Methods In the Stipa breviflora desert steppe research area, BSNE collecting sand boxes were set in the randomly distributed paddock experiment sites for 11 year with different grazing intensities $(0.15 、 0.30 、 0.45 、 0$ sheep $\cdot \mathrm{hm}^{-2} \cdot \mathrm{month}^{-1}$, corresponding to light grazing LG, moderate grazing MG, heavy grazing HG and control CK, respectively). The quantitative relationship between grazing intensity and sediment flux, and the characteristics of sediment soil particle were conducted in four sampling periods through 2 years (April 2013 to April 2015).

Important findings (1) Grazing intensity had a significant effect on the sediment flux $(p<0.05)$, and the sediment flux increased with the increase of grazing intensity. The response of sediment flux to grazing intensity was

收稿日期Received: 2016-01-22 接受日期Accepted: 2016-05-09

* 共同第一作者 Co-first author

** 通信作者Author for correspondence (E-mail: nmghanguodong@163.com) 
variable with season. The daily average sediment flux $\left(13.12 \mathrm{~g} \cdot \mathrm{m}^{-1} \cdot \mathrm{d}^{-1}\right)$ during the period of April to October was smaller than that from October to April $\left(18.74 \mathrm{~g} \cdot \mathrm{m}^{-1} \cdot \mathrm{d}^{-1}\right)$. The sediment flux difference of different grazing intensities was greater from April to October, with the 5 times daily average sand flux in the heavy grazing paddock that in the control. The average sediment flux difference of different grazing intensities was small from October to April. (2) The relationship between the natural logarithm of sediment flux at different height and the vertical height had a better binomial fitting from April to October, and there was no obvious regular pattern about flux vertical distribution from October to April, and the vertical flux difference of grazing intensities was mainly expressed in $0-50 \mathrm{~cm}$ layer. (3) Sand sediment particle $\leqslant 250 \mu \mathrm{m}$ accounted for more than $85 \%$ of the total sediment, the sand sediment particle of $\leqslant 50 \mu \mathrm{m}$ ) size was significantly enriched, and the enrichment ratio increased with the increase of vertical height. The enrichment ratio of 125-250 $\mu \mathrm{m}$ particle and 50-125 $\mu \mathrm{m}$ particle decreased with the increase of vertical height, and the enrichment ratio of 125-250 $\mu \mathrm{m}$ particle was smaller than that of 50-125 $\mu \mathrm{m}$ particle $(p<0.05)$. Therefore grazing intensity had different influence on the sand flux in Stipa breviflora desert steppe, the greater the grazing intensity, the heavier the wind erosion was, and the effect of grazing intensity on grassland was enhanced by wind erosion.

Key words grazing intensity; wind erosion; windblown sediments; sediment flux; desert steppe

Citation: Li YQ, Li ZG, Dong Z, Wang ZW, Qu ZQ, Han GD (2016). Effects of grazing intensity on windblown sediment mass flux and particle size distribution in the desert steppe of Nei Mongol, China. Chinese Journal of Plant Ecology, 40, 1003-1014. doi: 10.17521/cjpe.2016.0045

土壤风蚀(wind erosion)普遍存在于干旱半干旱 区, 影响 $1 / 3$ 以上的陆地面积(Pimentel \& Kounang, 1998; Li et al., 2009), 为空气沙尘提供物质来源(李 少昆等, 2008), 是下游风沙沉降区空气污染的重要 来源。在北美的圣地亚哥, 引起呼吸道疾病的 $\mathrm{PM}_{10}$ 污染物中, 有50\%来自土壤颗粒, 空气污染程度与 风暴频率有直接的关系 (Garciachevesich et al., 2014)。在我国, 沙尘是北方许多城市的重要季节性 污染物(李万元等, 2012)。风蚀造成异地污染的同时, 在风沙源区带走大量的土壤营养物质, 是荒漠化地 区土壤养分变异的重要驱动力(Li et al., 2007)。沙漠 化过程中的地表粗化、营养物质丧失以及植被退化 等都与土壤风蚀有直接或间接的关系。1952-2001 年的统计结果(李万元等, 2012)表明, 内蒙古中西部 的强尘沙暴几乎占我国北方沙尘暴总频数的 $2 / 3$ 。于 国茂等(2011)按照土壤风蚀危险度模型也将内蒙古 中西部地区的草原区域评定为风蚀危险型和风蚀强 险型。内蒙古中西部地区的草原类型主要是荒漠草 原, 是旱生性最强的草原生态系统, 承受着比其他 草原类型更为严重的风蚀和放牧双重胁迫(Aubault et al., 2015)。

风蚀率通常用风蚀模数, 即单位面积、单位时 间的土壤风蚀量来表示。普遍认为风沙流水平通量 $(Q)$ 和风蚀率存在定量关系( Li et al., 2007), 单位时 间、单位面积土壤沙尘释放 $F_{a}=K \times Q\left(F_{a}\right.$ 为垂直通
量, $K$ 为常数项)的关系被广泛认可(Shao et al., 1993), $Q$ 是风蚀研究中的关键环节(Basaran et al., 2011)。完整的植被有助于近地表土壤结构的稳定, 可以有效地减少风沙通量。在干旱、半干旱地区保 持一定临界植被盖度对控制土壤风蚀的发生与发展 具有重要的意义。在东北地区, 裸露的农田是重要 的沙尘来源。保护草原能有效地抑制沙尘排放 (Zhang et al., 2015)。植被抑制风蚀的效果可能存在 一个盖度阈值。由于风蚀对土壤、植被和气候变化 空间格局的敏感性, 量化影响风蚀速率的不同管理 措施非常困难(Aubault et al., 2015)。关于土壤风蚀 的研究结论大多通过理论推导或风洞模拟实验而获 得, 研究对象大多是沙漠分布区的风沙土(董智, 2004; 杨兴华等, 2013), 或者关注于农牧交错区耕 作措施对土壤风蚀的影响作用(李晓丽和申向东, 2006)。仅有少量研究关注放牧对草地风蚀的影响, 但侧重于单一放牧强度的水土流失强度和风沙流变 化等。不同放牧梯度下草原风蚀过程的野外系统观 测研究尚不多见。

短花针茅(Stipa breviflora)草原是内蒙古荒漠草 原的重要组成部分。放牧导致短花针茅草原退化、 沙化一直是内蒙古荒漠草原面临的主要环境问题。 针对荒漠草原生态系统, 学者们开展了较多放牧影 响的研究工作 (韩国栋等, 2007; 王忠武, 2009; Kemp et al., 2013), 但是迄今我们对放牧强度对荒 
漠草原风蚀特征的影响并不清楚。为此, 本文作者 依托内蒙古荒漠草原野外科学观测试验站建立的长 期放牧实验平台, 通过2年野外BSNE集沙仪取样, 开展了不同放牧强度对荒漠草原风蚀过程的影响研 究, 主要想回答两个方面的科学问题: (1)不同季节, 荒漠草原风沙通量对放牧强度如何响应? (2)荒漠 草原风沙沉积物颗粒粒径的主要分布特征及其对不 同放牧强度的响应有何不同?

\section{1 材料和方法}

\section{1 试验地概况}

试验地位于内蒙古自治区乌兰察布市四子王旗 王府一队 $\left(41.79^{\circ} \mathrm{N}, 111.90^{\circ} \mathrm{E}\right.$, 海拔 $\left.1450 \mathrm{~m}\right)$, 这里 春季干旱多风, 夏季炎热, 年降水量 $311.4 \mathrm{~mm}$, 降 水主要集中在6-9月，6-9月降水量占全年降水量的 $70 \%$ 以上, 年平均气温 $3.4{ }^{\circ} \mathrm{C}$, 土壤类型为栗钻土。 群落类型为短花针茅 + 冷蒿 (Artemisia frigida)+无芒 隐子草(Cleistogenes songorica)。植物群落主要由20 多种植物组成, 建群种为短花针茅, 优势种为冷蒿 和无芒隐子草。试验区草地在2004年随机区组放牧 试验前一直沿用简单的连续放牧制度, 并且放牧牲 畜超过了草地载畜能力, 多年的过度利用使这里的 草地受到严重破坏, 草地生产力低下。由于气候干 旱和强烈的人为活动, 在围栏放牧之初试验区草地 已出现了一定程度的退化。

\section{2 试验设计}

放牧控制实验平台于2004年6月开始运行。该平 台占地面积约 $50 \mathrm{hm}^{2}$ 。实验处理采用随机区组放牧 试验, 放牧区共分成3个区组, 每个区组内设有 3 个 载畜率水平和一个不放牧的对照, 分别为轻度放牧 (LG)、中度放牧 $(\mathrm{MG})$ 、重度放牧(HG) 和对照 $(\mathrm{CK}) 4$ 个处理, 随机排列。每个处理设为3次重复(LG1、

LG2、LG3; MG1、MG2、MG3; HG1、HG2、HG3; $\mathrm{CK} 1 、 \mathrm{CK} 2 、 \mathrm{CK} 3)$, 每个放牧小区面积为 $4.4 \mathrm{hm}^{2}$, 共 设12个放牧小区。家畜类型为绵羊, 放牧只数分别 为 $0 、 4 、 8 、 12$ 只, 对应的载畜率分别为: $0(\mathrm{CK})$ 、 0.15 (LG)、0.30 (MG)、0.45 (HG) sheep $\cdot \mathrm{hm}^{-2}$. month $^{-1}$ 。每年6-12月为放牧期。期间每天将家畜赶 入放牧区让其自由采食, 夜间赶回畜圈休息。

\section{3 取样和测定方法}

在每个放牧小区中间, 选择能代表对应放牧强 度的典型地段, 布置 $5 \mathrm{~m} \times 20 \mathrm{~m}$ 的小样区, 用于安
装BSNE沙尘采样器。每套BSNE沙尘采样器由不同 高度的取样器构成(Fryrear \& Saleh, 1993), 取样器 配有风叶和可旋转轴心, 安装在支撑杆的不同高 度; 采样器的高度可以调整。每个采样器集沙口宽2 $\mathrm{cm}$, 高 $5 \mathrm{~cm}$ 。含沙风进入集沙口后, 沙粒被捕获, BSNE沙尘采样器的集沙效率在 $90 \%$ 以上(Shao et al., 1993)。BSNE沙尘采样器按照 $4 \mathrm{~m}$ 间隔沿线安置 在每个 $5 \mathrm{~m} \times 20 \mathrm{~m}$ 小样区, 每个小区布置 4 套BSNE 沙尘采样器, 排列与盛行风垂直。每套系统(BSNE 沙尘采样器+中心支撑金属管)的中心支撑金属管被 垂直钉入地面以下至少 $50 \mathrm{~cm}$, 保证在实验期间不 发生沉降。4套BSNE沙尘采样器, 除1根1.5 m高的 BSNE沙尘采样器支撑杆安装 6 个高度集沙盒 $(0.1$ $\mathrm{m} 、 0.3 \mathrm{~m} 、 0.5 \mathrm{~m} 、 0.7 \mathrm{~m} 、 1.0 \mathrm{~m} 、 1.2 \mathrm{~m})$ 外, 其他 3 个 $1.2 \mathrm{~m}$ 高的支撑杆安装 3 个不同高度集沙盒 $(0.1 \mathrm{~m}$ 、 $0.5 \mathrm{~m} 、 1.0 \mathrm{~m})$, 每个小区15个集沙盒, 12 个小区, 共 180 个集沙盒。

BSNE沙尘采样器+中心支撑金属管在2013年 春季风季开始之前安装完毕, 全部集沙时间包含两 个完整年度，分别是2013年4月-2014年4月和2014 年4月-2015年4月 (表示为2013-2014年和2014-2015 年, 下同)。每年两次取样, 时间在10月中旬(采样期 4-10月, 对应于生长季, 下同)和次年4月中旬(采样 期上年10月至当年4月，对应于非生长季，下同), 每次取样时准确记录日期, 两次间隔日期用于计算 日平均通量(4次取样, 间隔日期分别是 $191 \mathrm{~d} 、 198$ d、 $187 \mathrm{~d} 、 176 \mathrm{~d}$ ); 由于第一个采集期集沙盒掉落地 面(可能是风或家畜触动导致), 在2013年10月共收 集了 178 个沙尘样品; 重新固定集沙盒后, 后期每 次取样获得 180 个样品, 4次取样, 总共采集 710 个沙 尘样品。每次取样时, 在 $5 \mathrm{~m} \times 20 \mathrm{~m}$ 小样区内采集土 壤表层和结皮样品。每次取 20 个土壤表层样品(0-5 $\mathrm{cm}), 12$ 个小区共 240 个样品; 4 次取样共 480 个土壤 样品。安装BSNE沙尘采样器的同时, 在CK2区安装 野外小型气象站(AG1000农田草地小气候站, 北京 天诺基业科技有限公司，北京)，用于测定试验期间 温度、降水量、空气湿度、土壤温/湿度、 $2.5 \mathrm{~m}$ 高 的风速风向、太阳辐射参数等指标, 所有气象指标 每10 min测定一次。

\section{4 计算和统计分析}

风沙流水平通量 $(Q)$ 可以用单位时间内垂直于 风向的固定高度单位宽度的风沙质量来表示, $Q$ 由 
不同高度风沙流 $q(\mathrm{z})$ 构成, $q(\mathrm{z})$ 有不同的拟合方程 (Fryrear \& Saleh, 1993)。本试验按照Shao和Raupach (1992)的方法进行拟合, 不同高度单位面积水平通 量 $q(\mathrm{z})$ 满足下面的方程(1)关系:

$$
q(z)=c \mathrm{e}^{\left(a z^{2}+b z\right)}
$$

这里 $a 、 b$ 和 $c$ 是拟合参数, $z$ 是集沙口高度 $(\mathrm{m})$ 。先将 方程两边取自然对数, 即 $\ln q(z)=a z^{2}+b z+\ln c$, 上述公式可以通过SPSS进行多项式拟合。通过二次 多项式函数建立不同垂直高度水平通量 $q$ 与集沙口 高度 $z$ 的关系; 通过拟合的多项式方程求得常数项 $a 、 b$ 和 $c$, 完成 $q(z)$ 方程的建立。

需要进一步对 $q(z)$ 方程进行从地表到 $1 \mathrm{~m}$ 高度 的积分计算求得 $Q$ 。根据积分方程(2)可以求得 $1 \mathrm{~m}$ 高度 $1 \mathrm{~m}$ 宽度截面范围内的水平通量 $Q$ 。

$$
Q=\int_{0}^{1 \mathrm{~m}} q(z) d z
$$

用Excel 2003和Origin 9.0软件构建图表, 采用 Matlab进行积分计算。采用IBM SPSS V21.0软件对 不同载畜率草原的风沙通量差异进行单因素方差分 析, 多重比较采用最小显著差异法 $(L S D)$ 进行, $p<$ 0.05 差异显著, 参数以平均值 \pm 标准偏差 (mean \pm $S D$ )表示。

\section{2 结果和分析}

\section{1 两个采样年度气象要素特征}

研究区2013-2014年和2014-2015年两个采样年 度总降水量分别为 $289 \mathrm{~mm}$ 和 $210 \mathrm{~mm}$, 降水量年度
差异较大，分别是多年平均降水量的 $91 \%$ 和 $69 \%$ (图 1); 按照生长季和非生长季进行划分, 2013-2014年 生长季和非生长季降水量分别为 $266.9 \mathrm{~mm}$ 和 22.1 $\mathrm{mm}$, 分别比2014-2015年对应时期高 $71.4 \mathrm{~mm}$ 和 $7.6 \mathrm{~mm}$; 生长季降水量差异主要表现在6-8月, 2013 年6-8月降水总量比2014年同期高120 mm。由于大 气相对湿度和降水量的相关性, 大气湿度变化与降 水量有类似的变化规律。

两个采样年度主导风向季节变化特征基本类似 (图2)，4-10月以西南风向为主，10月至次年4月以西 风为主; 2013-2014年大于 $7 \mathrm{~m} \cdot \mathrm{s}^{-1}$ 的总时间为 3803 $\mathrm{h}, 2014-2015$ 年为3 $798 \mathrm{~h}$, 分别占到对应时段的 $42 \%$ 和 $44 \%$ 。但大于 $7 \mathrm{~m} \cdot \mathrm{s}^{-1}$ 风速覆盖时段在季节间 存在差异(图2), 2013-2014年生长季平均风速大于7 $\mathrm{m} \cdot \mathrm{s}^{-1}$ 的时段达到 $2292 \mathrm{~h}$, 占到总时段的 $50 \%$, 而非 生长季大于 $7 \mathrm{~m} \cdot \mathrm{s}^{-1}$ 的时段共计 $1611 \mathrm{~h}$, 占统计时段 的34\%；2014-2015年的生长季和非生长季, 大于7 $\mathrm{m} \cdot \mathrm{s}^{-1}$ 风速段大约占对应时段的比例都是 $43 \%$ 。

\section{2 风沙流通量变化}

根据方程(2)计算的不同放牧强度水平输沙通 量见图3。年度、季节、载畜率的多因素单变量方差 分析(年度和季节间风沙通量差异采用 $t$ 检验方法)表 明载畜率和季节变化对平均风沙通量影响达到极显 著水平 $(p<0.05)$, 并且二者之间的交互效应对通量 也有显著影响 $(p<0.05)$; 随放牧强度增加, 风沙通 量呈显著增加趋势, LG、MG、HG的风沙通量分别 是对照区的1.4、2.2和2.5倍。多重比较表明, 两年的

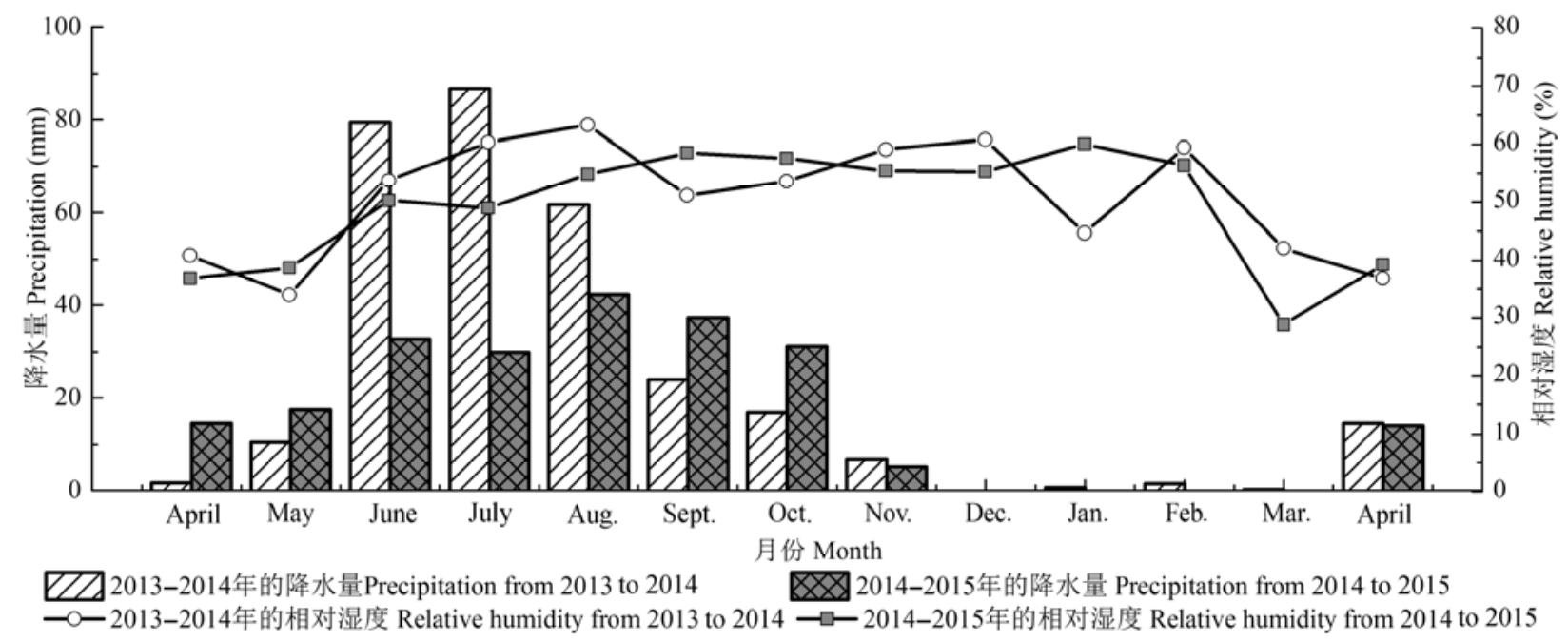

图1 试验站2013年4月至2015年4月降水量和相对湿度的月变化。

Fig. 1 Monthly variation of precipitation and relative humidity from April 2013 to April 2015 at the experimental site. 

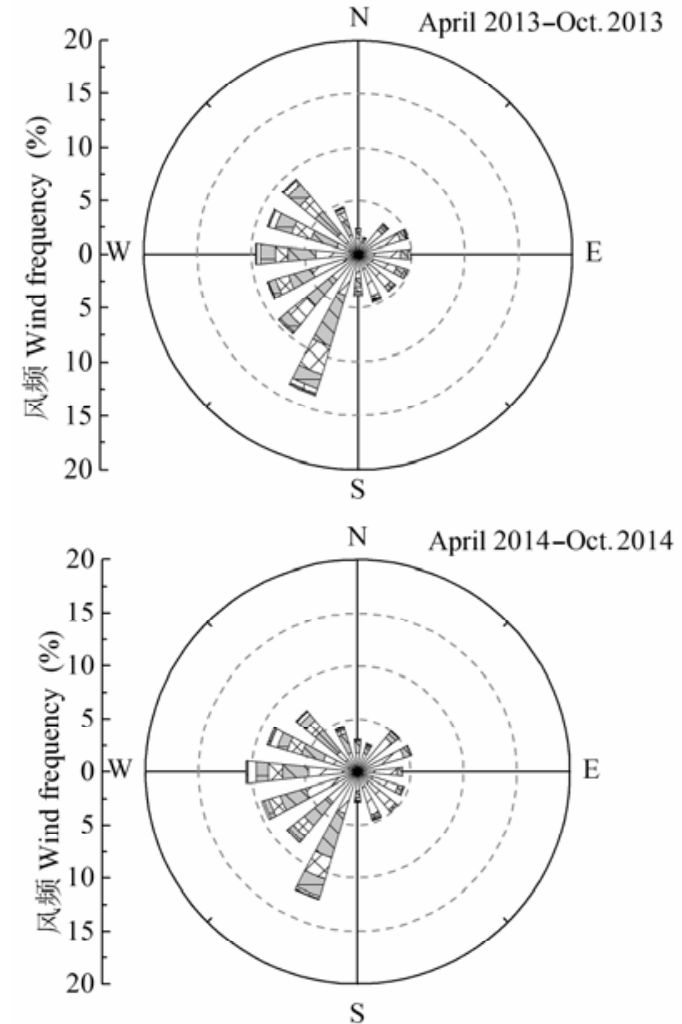
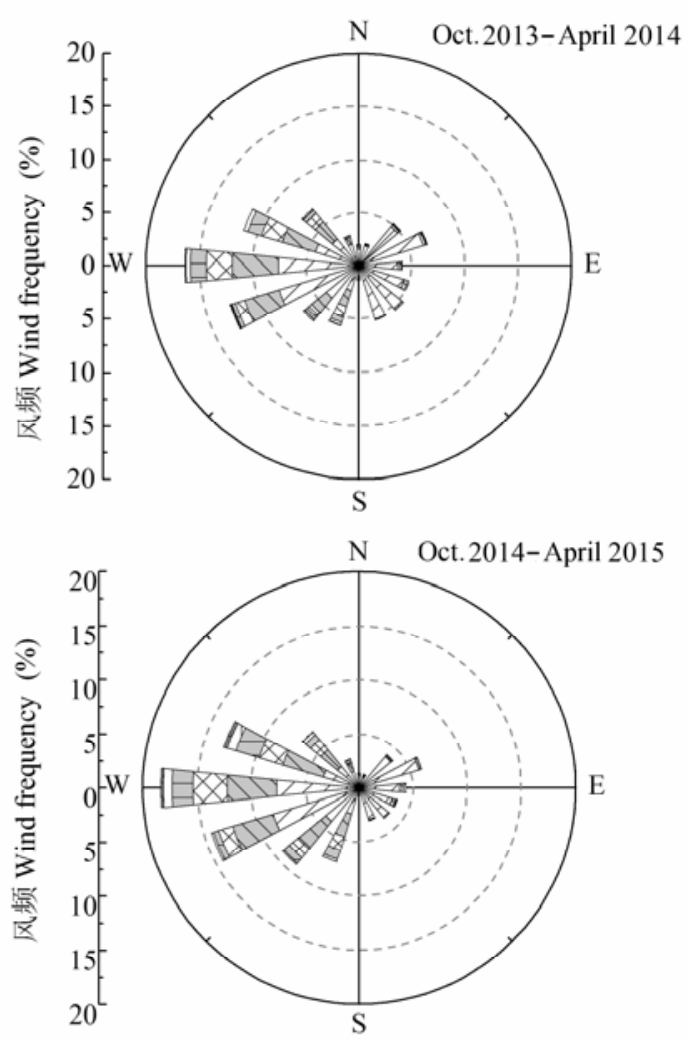

图2 不同采样期(2013年4月至2015年4月)风向风速分布。

Fig. 2 Distribution of frequency (\%) of wind speed from different directions in different sampling dates (April 2013 to April 2015).
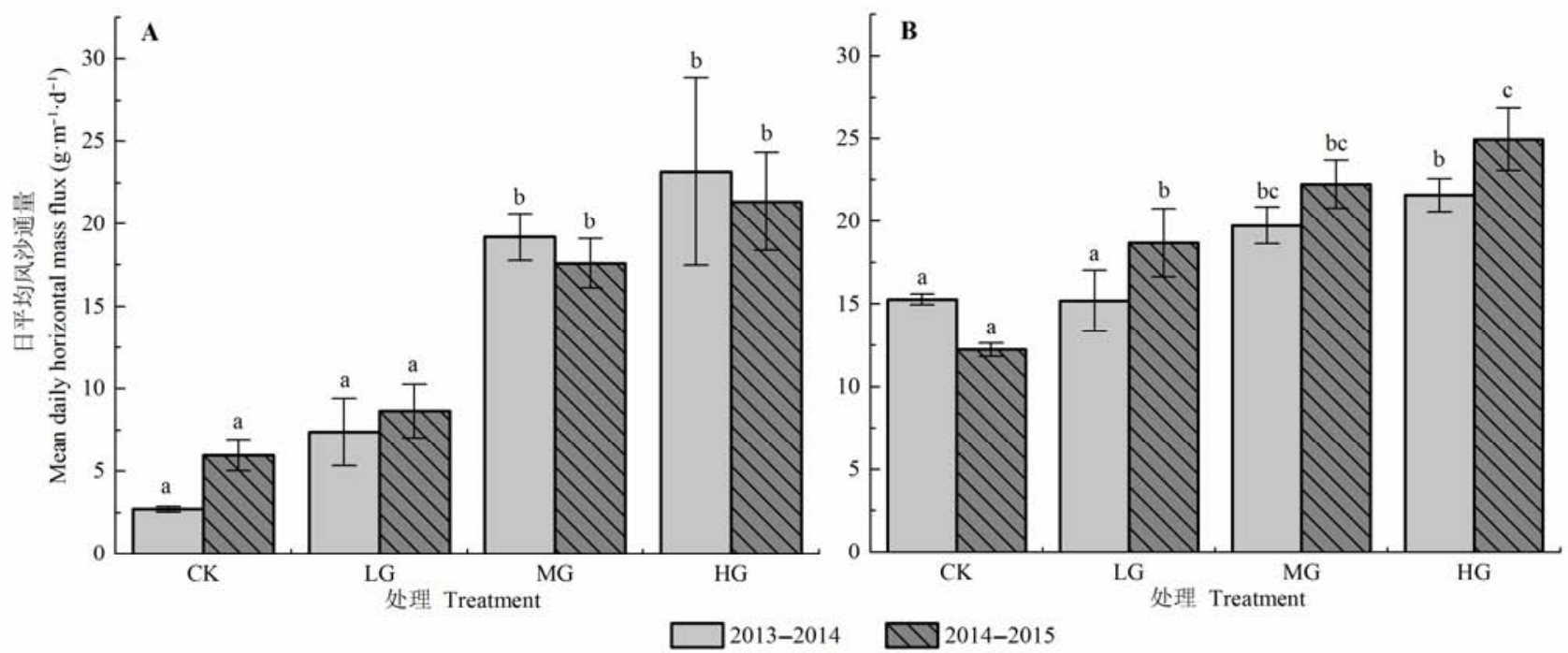

图3 不同放牧强度在生长季和非生长季日平均风沙通量变化(平均值沶准误差, $n=3$ )。 $\mathbf{A}$, 生长季。 $\mathbf{B}$, 非生长季。 CK, 对 照; LG，轻度放牧; MG，中度放牧; HG，重度放牧。不同小写字母表示同一时期不同放牧处理间差异显著 $(p<0.05)$ 。

Fig. 3 Mean horizontal mass flux versus different levels of stocking rate in growing-season and non-growing-season in two sampling years (mean $\pm S E, n=3$ ). A, Growing-season. B, Non-growing season. CK, control; LG, lightly grazed; MG, moderately grazed; HG, heavily grazed. Different lowercase letters indicate significant differences among the differently stocking rate during the same period $(p<0.05)$.

HG 和MG显著高于CK和LG $(p<0.05)$ ，其他处理间 无显著差异。非生长季平均风沙通量为 $18.74 \mathrm{~g} \cdot \mathrm{m}^{-1}$. $\mathrm{d}^{-1}$, 是生长季 $\left(13.12 \mathrm{~g} \cdot \mathrm{m}^{-1} \cdot \mathrm{d}^{-1}\right)$ 的1.4倍。风沙通量对
放牧强度的响应存在季节差异; 在生长季(4-10月) 风沙通量随放牧强度增强而增加的趋势比非生长季 (10月至次年4月)更明显(图3), HG区风沙通量是对 
照区的5倍，而非生长季不同放牧强度间风沙通量 差异缩小, HG区仅是对照区的1.7倍；2014-2015年 日平均风沙通量 $16.46 \mathrm{~g} \cdot \mathrm{m}^{-1} \cdot \mathrm{d}^{-1}$, 稍高于2013-2014 年的 $15.53 \mathrm{~g} \cdot \mathrm{m}^{-1} \cdot \mathrm{d}^{-1}$, 但年度间差异不显著 $(p=$
$0.378)$ 。

\section{3 不同放牧强度风沙流结构}

风沙通量自然对数与集沙口高度多项式拟合曲 线、方程和参数见图 4 。生长季的拟合效果 $\left(R^{2}\right.$ 平均
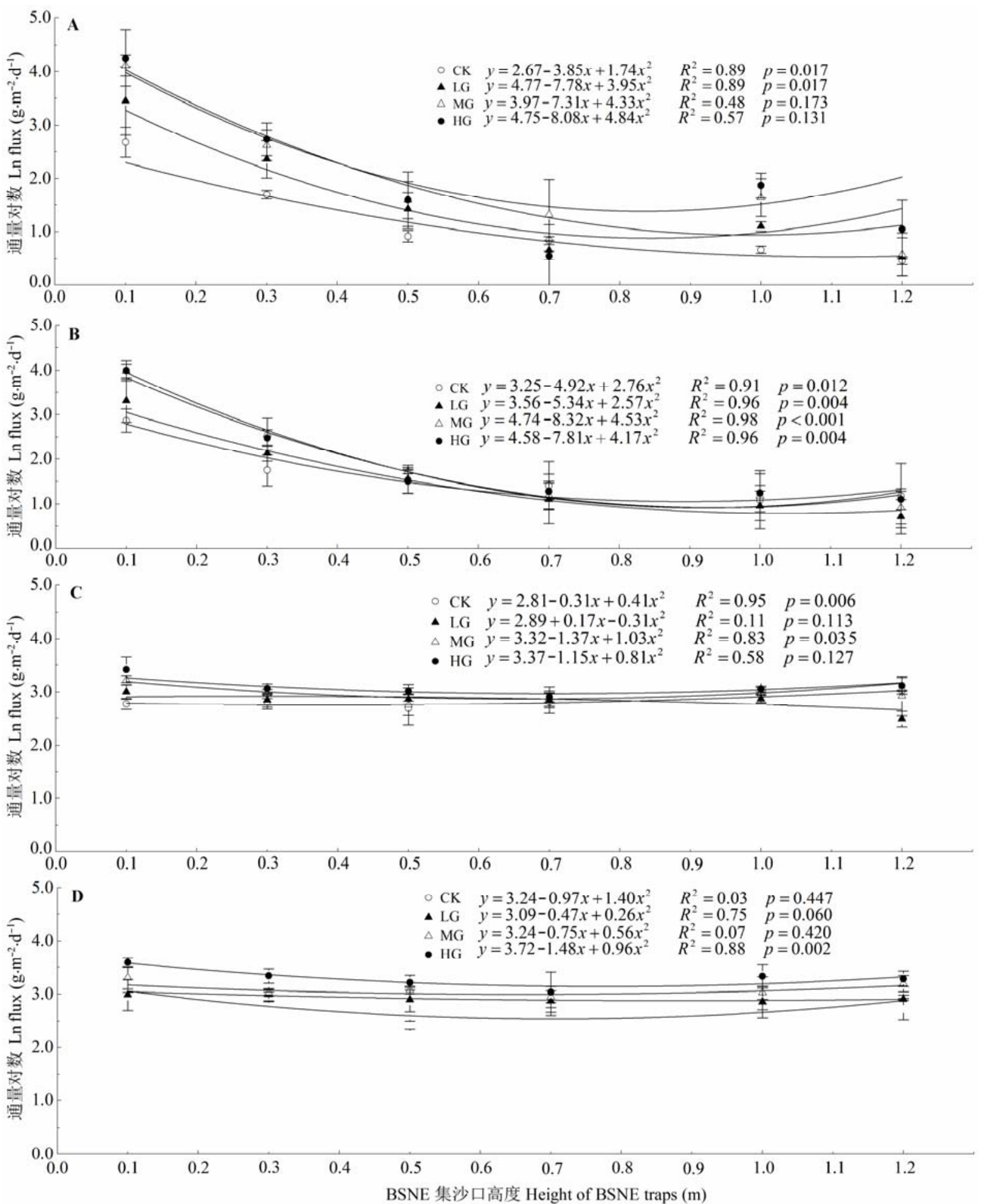

图4 不同采样期风沙流水平通量对数随高度的变化。A, 2013-2014年生长季。 B, 2014-2015年生长季。C, 2013-2014年非生 长季。D, 2014-2015年非生长季。CK, 对照; LG, 轻度放牧; MG 中度放牧; HG, 重度放牧。

Fig. 4 Natural logarithm of sediment flux as a function of height during different sampling seasons. A, 2013-2014 growing-season. B, 2014-2015 growing-season. C, 2013-1014 non-growing-season. D, 2014-2015 non-growing-season. CK, control; LG, lightly grazed; MG, moderately grazed; HG, heavily grazed. 
值0.830)好于非生长季 $\left(R^{2}\right.$ 平均值0.525)。拟合方程参 数统计表明, 特别是2014-2015年的生长季, 各个处 理的回归方程均达到显著水平 $(p<0.05)$, 在MG区 达到极显著水平 $(p<0.001)$; 而非生长季, 风沙通量 对数曲线随垂直高度变化变得更为平缓, 绝大多数 处理拟合方程未达到显著水平 $(p>0.05)$ 。在不同放 牧强度处理下，拟合曲线的相对位置不同，在0-50 $\mathrm{cm}$ 高度, 从下至上曲线依次为 $\mathrm{CK} 、 \mathrm{LG} 、 \mathrm{MG} 、 \mathrm{HG}$ 。 总体来看, 风沙通量自然对数和高度的二次拟合关 系在生长季的效果更好, 在植被较好的季节, 风沙 流结构有更显著的垂直梯度变化(图4), 而在非生长 季, Shao和Raupach (1992)的风沙通量指数递减方程
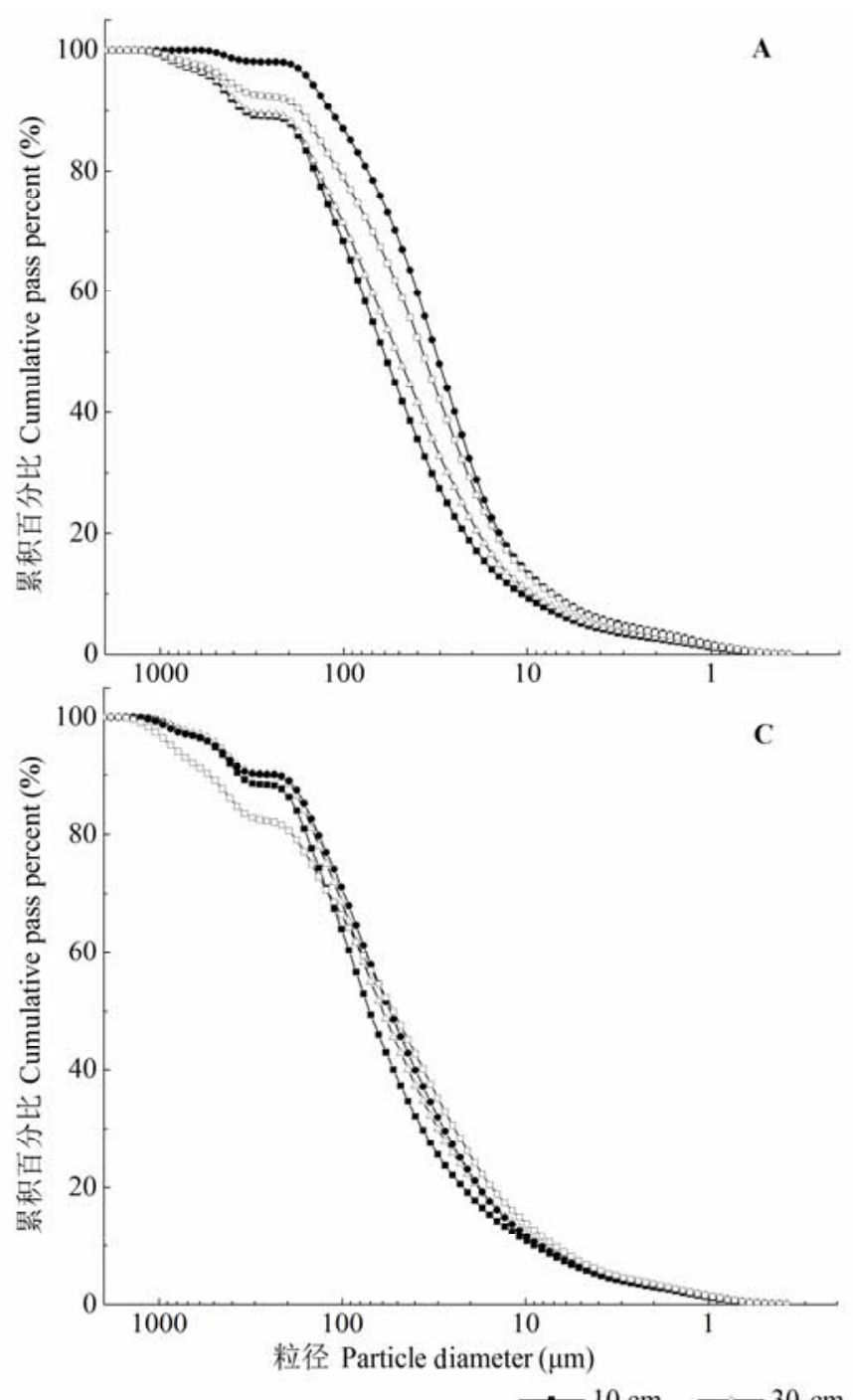

拟合效果有限。

\section{4 不同放牧强度风沙沉积物粒径特征}

为了便于分析, 选取两年度生长季对照区和 HG区不同高度 $(10 \mathrm{~cm} 、 30 \mathrm{~cm} 、 50 \mathrm{~cm} 、 100 \mathrm{~cm})$ 沉积 物粒径特征进行比较(图5)。对照区和HG区风沙沉 积物所有样品大于 $250 \mu \mathrm{m}$ 的粗砂粒含量均未超过 $15 \%$; 对照区10 cm高度集沙口捕获的风沙沉积物 中大于 $250 \mu \mathrm{m}$ 的粗砂粒平均含量为 $10.91 \%$, 而在 $100 \mathrm{~cm}$ 高度集沙口处, 大于 $250 \mu \mathrm{m}$ 的粗砂粒含量下 降为 $7.57 \%$, 垂直高度对大于 $250 \mu \mathrm{m}$ 的粗砂粒含量 影响显著 $(p<0.05)$; 在HG区，不同高度的大于 250 $\mu \mathrm{m}$ 的粗砂粒含量差异不显著 $(p>0.05)$, 平均含量
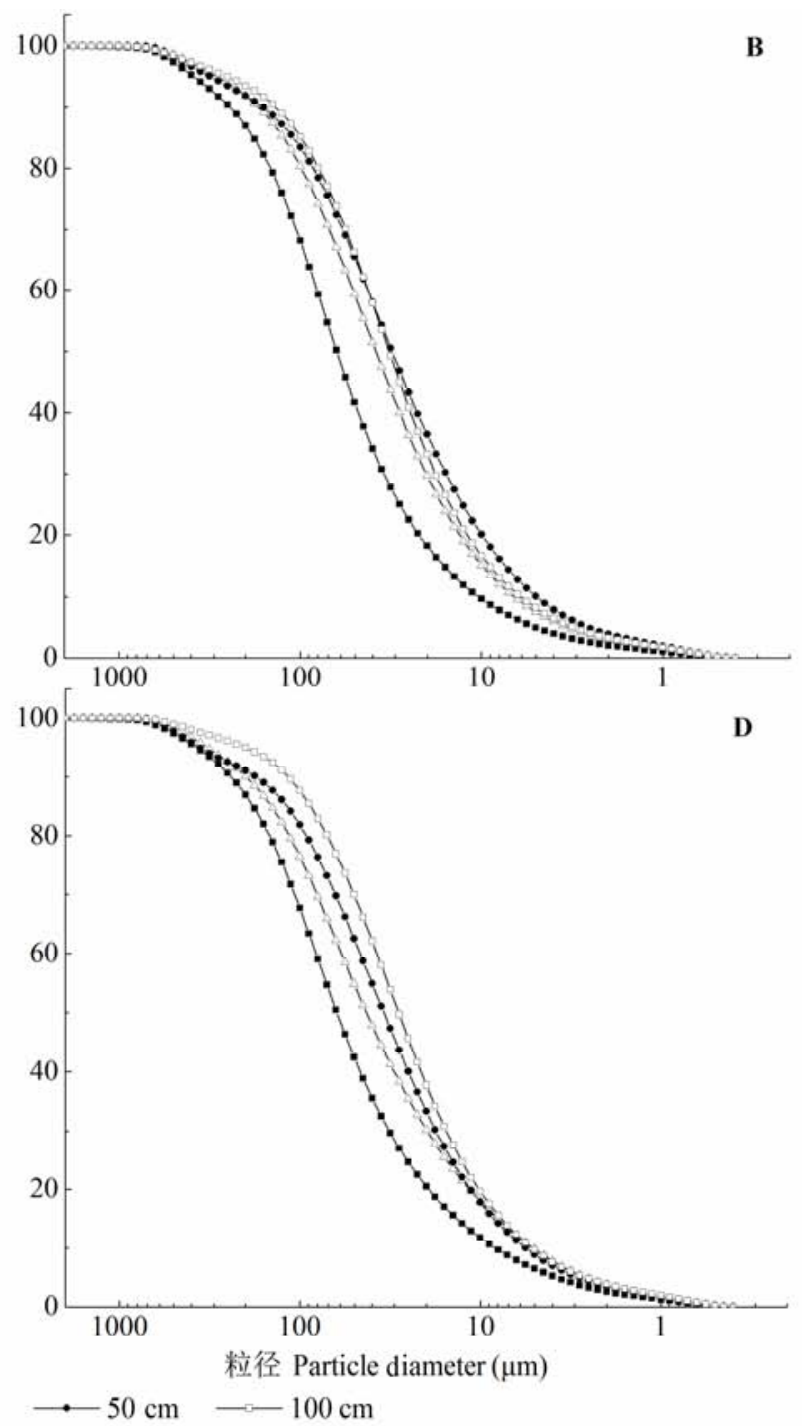

图5 对照(CK)和重度放牧(HG)区生长季(10月采样期)不同集沙高度的风沙沉积物粒径分布曲线。A, 2013年10月 CK区。B, 2014年10月CK区。C, 2013年10月HG区。D, 2014年10月HG区。10 cm、30 cm、50 cm、100 cm为集沙高度。

Fig. 5 Particle size distributions for sediment samples of the BSNE different heights on control (CK) and heavily grazed (HG) treatments during growing seasons (October sampling period). A, October 2013 CK. B, October 2014 CK. C, October 2013 HG. D, October 2014 HG. $10 \mathrm{~cm}, 30 \mathrm{~cm}, 50 \mathrm{~cm}$, and $100 \mathrm{~cm}$ indicate heights of the traps. 
为 $14.62 \%$ 。这表明HG区的风沙沉积物中粗颗粒含 量更多。小于 $50 \mu \mathrm{m}$ 的粉粒和黏粒含量在不同放牧 强度和垂直高度也有明显的变化：对照区10 $\mathrm{cm}$ 高 度的平均含量为 $44.9 \%$, 而在 $100 \mathrm{~cm}$ 高度平均含量 为 $61.0 \%$, 但随着高度增加, 小于50 $\mu \mathrm{m}$ 的粉粒和黏 粒含量并非线性增加; 对照区含量峰值出现在50 $\mathrm{cm}$ 高度, 达到70.20\%, 显著高于其他垂直高度的细 颗粒含量; HG区的细颗粒含量从近地表 $10 \mathrm{~cm}$ 高度 的 $40.02 \%$ 增加到 $100 \mathrm{~cm}$ 高度的 $49.92 \%$, 细颗粒峰值 出现在70 $\mathrm{cm}$ 高度(59.89\%)。这表明HG区风沙沉积 物中细颗粒含量普遍较低, 且随垂直高度的增加而 变化的幅度较小。
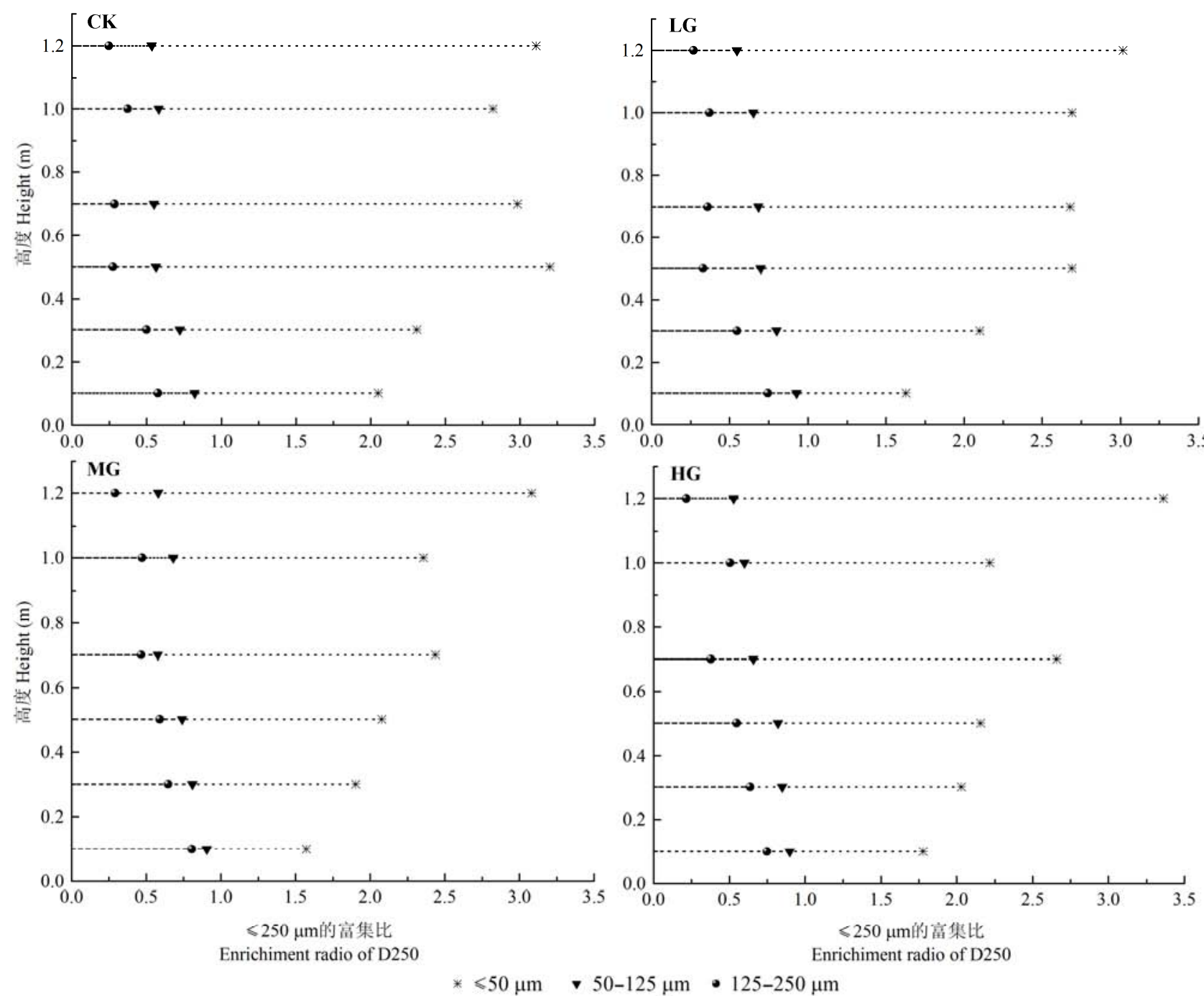

图6 不同放牧处理风沙沉积物中直径 $(D) \leqslant 250 \mu \mathrm{m}$ 颗粒富集比随高度的变化(富集比=沉积物中某粒径含量/土壤表土中对 应粒径含量)。CK, 对照。LG, 轻度放牧。MG, 中度放牧。HG, 重度放牧。

Fig. 6 Enrichment factor (E) of particle size $\leqslant 250 \mu \mathrm{m}$ in sediments versus different heights of different treatments (enrichment factor = content of particle size in the sediments / content of the relative particle size in the surface soil layer). CK, Control. LG, Lightly grazed. MG, Moderately grazed. HG, Heavily grazed. D250, particle size $\leqslant 250 \mu \mathrm{m}$. 
稳定, 而 $C K$ 和 $L G$ 中, $50 \mathrm{~cm}$ 高度以上的 $E$ 值随高度 变化较小, $C K$ 和 $L G$ 的平均 $E$ 值分别为 3.02 和 2.77 。在 $D \leqslant 250 \mu \mathrm{m}$ 颗粒的 3 个分级中, $E$ 值最小的是 125-250 $\mu \mathrm{m}$ 颗粒, 平均值为 $0.47,50-125 \mu \mathrm{m}$ 颗粒为 $0.70 ; 125-250 \mu \mathrm{m}$ 和 $50-125 \mu \mathrm{m}$ 颗粒的 $E$ 值呈现基本 与 $D \leqslant 50 \mu \mathrm{m}$ 颗粒相反的变化趋势, 随高度增加, $E$ 值逐渐减小, 但变化幅度较小。

\section{3 讨论}

\section{1 放牧强度对输沙通量的影响}

风蚀率是风蚀研究中最受关注的问题(杜鹏飞 和刘孝盈, 2012), 包括利用WEPS (Wagner, 2013)、

WEAM (Shao et al., 1996)等不同风蚀预报模型的风 蚀量估测(邹学勇等, 2014)、遥感和GIS技术的风蚀 动态监测技术(Santini et al., 2010)、元素示踪技术 等。由于尺度效应和因子的复杂性(Webb et al., 2014), 在判断具体土地利用模式或者小尺度风蚀 强度时, 各种野外实测技术如风蚀盘法和集沙仪等 (Fryrear, 1986)仍是风蚀率测定常用的方法。由于风 蚀盘法容器材料和土壤性质的差异, 不能完全反映 野外土壤复杂的表面状况, 只适合于单次风蚀事件 的风蚀量估测。武川县试验站采用风蚀盘法测得 10 月至次年4月的裸地风蚀量(赵彩霞等, 2005)达到 $3700 \mathrm{t} \cdot \mathrm{km}^{-2}$, 明显高于相同区域的其他研究结果 (孙悦超等, 2007); 集沙仪法测定输沙通量是风沙流 结构研究中最常用的方法。许多研究者开发了不同 类型的集沙仪, BSNE是近年来用于野外测定风蚀 过程常用的集沙仪类型。风蚀作为一个自然过程, 涉及风蚀因子的许多方面, 但土地利用模式对各种 风蚀因子的影响是人类管理生态系统风蚀变化的主 要原因(邹学勇等, 2014)。放牧导致草地植被盖度降 低是影响风沙通量的主要原因之一。在非洲的 Kalahari荒漠(Wiggs et al., 1994), 由于放牧等原因 降低植被盖度 $60 \%$ 时, 近地表风速增加 $200 \%$; 放牧 通过减少植被和破坏土壤表皮, 加快风力侵蚀速度 (Belnap et al., 2009)。

本试验所在的长期放牧样地, 从2004年开始实 施随机区组放牧试验, 最初不同载畜率间的植被盖 度没有明显不同, 但从2007年开始, MG和HG区的 盖度已经发生明显变化, 显著低于CK和LG区, 放 牧对盖度的累积效应在第三年开始得到体现(王忠 武, 2009)。两个采样年度的非生长季平均风沙通量
比生长季高 $41 \%$, 反映了植被盖度季节变化导致的 风蚀强度的变化。与CK相比, 生长季的HG区平均 风沙通量增加5.1倍; 非生长季, 荒漠草原植物盖度 普遍比较低, 在风速条件大致相同的条件下(图2), 各放牧处理的风沙通量都比生长季有明显的增加, 但CK区风沙通量和HG区风沙通量差距缩小。这表 明放牧对风沙通量的影响强度与不同季节的降水、 盖度等因子有关。整个试验期HG区的平均风沙通量 为22.76 $\mathrm{g} \cdot \mathrm{m}^{-1} \cdot \mathrm{d}^{-1}$ 。与 $\mathrm{CK}$ 相比, $\mathrm{HG}$ 导致风沙通量增 加2.5倍(图3)。植被盖度和输沙通量之间不是简单的 线性关系(Liu \& Westphal, 2001), 植被盖度作为重 要的风蚀影响因子, 也影响着近地表风速、气流和 土壤的接触面积、土壤水分等其他风蚀影响因子 (李 万元等, 2007), 植被盖度增加有效地降低了土壤对 风蚀的敏感性(Shabani et al., 2014)。荒漠草原非生 长季不同放牧强度间的盖度差异比生长季间的盖度 差异要小。

\section{2 风沙流结构和风沙沉积物粒度特征}

许多学者在风洞或野外都进行过风沙流结构特 征的研究。Shao和Raupach (1992)、Zobeck和Stout (1996)、Namikas (2003)的拟合结果表明, 指数函数 能够较好地模拟近地表风沙流结构特征。但风沙流 垂直结构变化受沙粒粒径、沙源、下垫面特征、风 速等因素的影响, 野外过程比风洞复杂得多, 即使 是风洞试验, 结果也有较大差异。Dong等(2006)开 展的风洞实验发现, 近床面附近某一高度以下的输 沙量小于指数分布预测值; 而 $\mathrm{Ni}$ 等(2003)的风洞实 验结果是近床面输沙量相对于指数函数的计算发生 正偏差。由此可见, 不同研究者对输沙量垂向分布 规律的认识存在争议, 输沙量垂直分布规律的研究 结果大致可以分为三种类型: 完全符合指数递减分 布、上部分符合指数递减而下部分偏小、上部分符 合指数递减而下部分偏大。本研究中, 不同季节风 沙流垂向分布的指数拟合方程拟合效果不同: 生长 季(4-10月)由于有较好的植被覆盖, 不同垂直高度 风速存在明显不同, 风沙流通量指数递减趋势比较 明显; 而非生长季(10月至次年4月), 荒漠草原处于 枯黄期, 植被盖度普遍偏低, 垂直高度间风速差异 较小, 而不同气流强度携带的风蚀颗粒达到的高度 也有很大不同, 导致不同垂直高度间风沙流通量累 计量差异减小, 垂直分布曲线表现更为平缓。根据 拟合曲线的相对位置可以看出, 在0-50 cm高度, 拟 
合曲线从下到上依次为 $C K 、 L G 、 M G 、 H G$, 表明载 畜率增加, 主要增强了 0-50 cm层次的通量分配。

Lyles和Tararko (1986)在美国Kansas草原研究 发现, 36年的风蚀作用使得表层土壤砂粒含量上升 $6.5 \%$, 黏粒含量下降 $7.2 \%$ 。在澳大利亚南部的牧场 也发现, 在20周内风蚀可以使土壤中大于 $250 \mu \mathrm{m}$ 颗 粒的含量增加, 75-210 $\mu \mathrm{m}$ 和小于 $2 \mu \mathrm{m}$ 颗粒的含量 减少(Leys \& Mctainsh, 1996)。土壤中大于 $250 \mu \mathrm{m}$ 颗 粒的含量逐渐增加, 这与本研究风沙沉积物中以小 于 $250 \mu \mathrm{m}$ 颗粒为主的特征一致; 但对于更精细范围 的50-125 $\mu \mathrm{m}$ 颗粒, 因为风蚀物中该粒径范围的富 集比小于1, 因此也属于风蚀不敏感颗粒。在本研究 中各粒径范围, 只是小于50 $\mu \mathrm{m}$ 的颗粒富集比大于 1 , 与澳大利亚南部牧场的敏感颗粒明显不同。也有 学者认为, 细颗粒损失量实际是风蚀和大气沉降的 净结果, 沉降本身会抵消一部分侵蚀造成的损失。 由于细颗粒含有更多的营养物质, 细颗粒损失可能 是导致草原养分枯竭、植被退化的一个重要机制(苏 永中和赵文智, 2005)。在本研究区附近阴山旱作耕 地风蚀颗粒研究(李晓丽和申向东, 2006)表明, 在裸 露耕地上, 大于 $2 \mathrm{~mm}$ 的颗粒粒径的百分含量占整 个颗粒粒径的 $32 \%$, 认为长期受风蚀影响, 地表已 经粗化。本研究各集沙盒的风沙沉积物中未收集到 大于 $2 \mathrm{~mm}$ 的颗粒, 小于 $250 \mu \mathrm{m}$ 的颗粒占到 $85 \%$, 放 牧草原与耕地有完全不同的风沙粒径结构。风沙沉 积物与土壤 $0-5 \mathrm{~cm}$ 粒径对比结果表明, 随着高度增 加, 风蚀物颗粒组成逐渐变细。小于 $250 \mu \mathrm{m}$ 风蚀颗 粒富集图(图5)表明, 风蚀作用下, 土壤表土中小于 $50 \mu \mathrm{m}$ 颗粒最为活跃, 而50-125 $\mu \mathrm{m}$ 和125-250 $\mu \mathrm{m}$ 颗粒在表土中有相应的聚集现象。

\section{4 结论}

放牧强度对内蒙古荒漠草原风蚀过程具有重要 影响, 风沙通量随放牧强度的增加呈逐渐增加的趋 势, 且风沙通量对放牧强度的响应受季节变化的影 响; 过度放牧导致草地植被高度和盖度降低、地表 裸露、地表破碎化。季节变化涉及植被、气候、地 表状况等的风蚀因子变化。荒漠草原非生长季平均 风沙通量显著高于生长季; 放牧强度间的风沙通量 差异主要表现在近地表层次。生长季的风沙流结构 垂向分布符合指数递减函数, 而非生长季, 指数递 减函数拟合效果较差; 内蒙古荒漠草原风沙沉积物
以直径小于 $250 \mu \mathrm{m}$ 的颗粒为主, 小于 $50 \mu \mathrm{m}$ 的粒径 在沉积物中存在明显的富集作用, 随高度增加富集 比逐渐增加; 放牧导致细颗粒随垂直高度增加而累 计。本文在 4 种载畜率条件下分析了荒漠草原不同放 牧利用强度对风沙通量和沉积物粒径的影响, 为不 同放牧情景下的荒漠草原风蚀模型建立提供了基础 数据和参考。放牧对植被盖度和侧影盖度(lateral coverage)、地表破碎度、土壤含水量等风蚀因子的 影响是导致风沙通量变化的直接原因。特别是放牧 作用下株从结构和群落结构决定的侧影盖度的变化 (Li et al., 2014)可能对风蚀过程的影响更大。在未来 研究中应更多地考虑放牧和株丛结构的耦合效应, 以便深入揭示荒漠草原风蚀过程对放牧管理的响 应。

基金项目 国家自然科学基金(31260123)、国家公 益行业专项计划项目(201003019和200903060)和教 育部草地资源重点实验室和创新团队支持项目。

\section{参考文献}

Aubault H, Webb NP, Strong C, Mctainsh GH, Leys JF, Scanlan JC (2015). Grazing impacts on the susceptibility of rangelands to wind erosion: The effects of stocking rate, stocking strategy and land condition. Aeolian Research, 17, 89-99.

Basaran M, Erpul G, Uzun O, Gabriels D (2011). Comparative efficiency testing for a newly designed cyclone type sediment trap for wind erosion measurements. Geomorphology, 130, 343-351.

Belnap J, Reynolds RL, Reheis MC, Phillips SL, Urban FE, Goldstein HL (2009). Sediment losses and gains across a gradient of livestock grazing and plant invasion in a cool, semi-arid grassland, Colorado Plateau, USA. Aeolian Research, 1, 27-43.

Dong Z (2004). Research on Farmland Wind-Sand Disaster of Oasis and Its Control Mechanism in Ulan Buh Desert. $\mathrm{PhD}$ dissertation, Beijing Forestry University, Beijing. 59-68. (in Chinese with English abstract). [董智 (2004). 乌兰布和沙漠绿洲农田沙害及其控制机理研究. 博士 学位论文, 北京林业大学, 北京. 59-68.]

Dong ZB, Qian GQ, Luo WY, Wang HT (2006). Analysis of the mass flux profiles of an aeolian saltating cloud. Journal of Geophysical Research, 111, 1-11.

Du PF, Liu XY (2012). A review of measurement of wind erosion rate in China research of soil and water conservation. Research of Soil and Water Conservation, 19, 275-281. (in Chinese with English abstract) [杜鹏飞, 刘孝盈 (2012). 中国土壤风蚀速率实测研究述评. 水土保持研 究, 19, 275-281.] 
Fryrear DW (1986). A field dust sampler. Journal of Soil and Water Conservation, 41, 117-120.

Fryrear DW, Saleh A (1993). Field wind erosion: Vertical distribution. Soil Science, 155, 230-294.

Garciachevesich P, Alvarado S, Neary DG, Valdes R, Valdes J, Aguirre JJ, Mena M, Pizarro R, Jofre P, Vera M, Olivares C (2014). Respiratory disease and particulate air pollution in Santiago Chile: Contribution of erosion particles from fine sediments. Environmental Pollution, 187, 202-205.

Han GD, Jiao SY, Biligetu, Aodenggaowa (2007). Effects of plant species diversity and productivity under different stocking rates in the Stipa breviflora Griseb. desert steppe. Acta Ecologica Sinica, 27, 182-188. (in Chinese with English abstract) [韩国栋, 焦树英, 毕力格图, 敖登高娃 (2007). 短花针茅草原不同载畜率对植物多样性和草地 生产力的影响. 生态学报, 27, 182-188.]

Kemp DR, Han GD, Hou XY, Michalk DL, Hou FJ, Wu JP, Zhang YJ (2013). Innovative grassland management systems for environmental and livelihood benefits. Proceedings of the National Academy of Sciences of the United States of America, 110, 8369-8374.

Leys J, Mctainsh GH (1996). Sediment fluxes and particle grain-size characteristics of wind-eroded sediments in southeastern Australia. Earth Surface Processes and Landforms, 21, 661-671.

Li J, Okin GS, Alvarez L, Epstein HE (2007). Quantitative effects of vegetation cover on wind erosion and soil nutrient loss in a desert grassland of southern New Mexico, USA. Biogeochemistry, 85, 317-332.

Li J, Okin GS, Epstein HE (2009). Effects of enhanced wind erosion on surface soil texture and characteristics of windblown sediments. Journal of Geophysical Research, 114, $1-8$.

Li J, Okin GS, Tatarko J, Webb NP, Herrick J (2014). Consistency of wind erosion assessments across land use and land cover types: A critical analysis. Aeolian Research, 15, 253-260.

Li SK, Lu M, Wang KR, Wang X (2008). Soil erosion of the main ground surface types influences on formation of dust storm in south Xinjiang. Scientia Agricultura Sinica, 41, 3158-3167. (in Chinese with English abstract) [李少昆, 路明, 王克如, 王旭 (2008). 南疆主要地表类型土壤风 蚀对形成沙尘暴天气的影响. 中国农业科学, 41, 31583167.]

Li WY, Dong ZB, Lü SH, Wang SG, Ao YH (2012). Spatial distribution and temporal tendency of the dust storm frequency in north and northwest China for the recent nearly half a century. Climate Change Research Letters, (1), 1-12. (in Chinese with English abstract) [李万元, 董治宝, 吕世华, 王式功, 奥银焕 (2012). 近半个世纪中国北方 沙尘暴的空间分布和时间变化规律回顾. 气候变化研 究快报, (1), 1-12.]
Li WY, Sheng ZB, Lü SH, Li YH (2007). Sensitivity tests of factors influencing wind erosion. Journal of Desert Research, 27, 78-87. (in Chinese with English abstract) [李 万元, 沈志宝, 吕世华, 李耀辉 (2007). 风蚀影响因子 的敏感性试验. 中国沙漠, 27, 78-87.]

Li XL, Shen XD (2006). Experimental study on the distribution characteristics of the saltation particle of aeolian sediment in bare tillage. Transactions of the CSAE, 22, 74-77. (in Chinese with English abstract) [李晓丽, 申向东 (2006). 裸露耕地土壤风蚀跃移颗粒分布特征的试验研究. 农 业工程学报, 22, 74-77.]

Liu M, Westphal DL (2001). A study of the sensitivity of simulated mineral dust production to model resolution. Journal of Geophysical Research, 106, 18099-18112.

Lyles L, Tararko J (1986). Wind erosion effects on soil texture and organic matter. Journal of Soil and Water Conservation, 41, 191-193.

Namikas S (2003). Field measurement and numerical modelling of aeolian mass flux distributions on a sandy beach. Sedimentology, 50, 303-326.

Ni JR, Li ZS, Mendoza C (2003). Vertical profiles of aeolian sand mass flux. Geomorphology, 49, 205-218.

Pimentel D, Kounang N (1998). Ecology of soil erosion in ecosystems. Ecosystems, 1, 416-426.

Santini M, Caccamo G, Laurenti A, Noce S, Valentini R (2010). A multi-component GIS framework for desertification risk assessment by an integrated index. Applied Geography, 30, 394-415.

Shabani F, Kumar L, Esmaeili A (2014). Improvement to the prediction of the USLE K factor. Geomorphology, 204, 229-234.

Shao Y, Raupach M (1992). The overshoot and equilibration of saltation. Journal of Geophysical Research, 97, 2055920564.

Shao Y, Raupach M, Findlater P (1993). Effect of saltation bombardment on the entrainment of dust by wind. Journal of Geophysical Research, 98, 12719-12726.

Shao Y, Raupach M, Leys J (1996). A model for predicting aeolian sand drift and dust entrainment on scales from paddock to region. Soil Research, 34, 309-342.

Su YZ, Zhao WZ (2005). Soil organic carbon dynamics: Wind erosion effect. Acta Ecologica Sinica, 25, 2049-2054. (in Chinese with English abstract) [苏永中, 赵文智 (2005). 土壤有机碳动态: 风蚀效应. 生态学报, 25, 2049-2054.]

Sun YC, Ma SS, Chen Z, Zhao YL, Sun YR (2007). Test and analysis of wind erosion of land surface soil in arid and semi-arid regions in north areas of Yinshan Mountain. Transactions of the CSAE, 23, 1-5. (in Chinese with English abstract) [孙悦超, 麻硕士, 陈智, 赵永来, 孙宇瑞 (2007). 阴山北麓干旱半干旱区地表土壤风蚀测试与分 析. 农业工程学报, 23, 1-5.]

Wagner LE (2013). A history of wind erosion prediction doi: 10.17521/cjpe.2016.0045 
models in the united states department of agriculture: The wind erosion prediction system (WEPS). Aeolian Research, 10, 9-24.

Wang ZW (2009). Effect of Stocking Rate on Ecosystem stability of Stipa brevilora Desert Steppe. $\mathrm{PhD}$ dissertation, Inner Mongolia Agricultural University, Hohhot. 5-35. (in Chinese) [王忠武 (2009). 载畜率对短花针茅荒漠草原 生态系统稳定性的影响. 博士学位论文, 内蒙古农业大 学, 呼和浩特. 5-35.]

Webb NP, Okin GS, Brown S (2014). The effect of roughness elements on wind erosion: The importance of surface shear stress distribution. Journal of Geophysical Research, 119, 6066-6084.

Wiggs GFS, Livingstone I, Thomas DSG, Bullard JE (1994). Effect of vegetation removal on airflow patterns and dune dynamics in the southwest Kalahari desert. Land Degradation and Development, 5, 13-24.

Yang XH, He Q, Ali M. Huo W, Liu XC (2013). Observational study on near-surface horizontal san-dust flux of sandstorms in the southeastern fringe of the Taklimakan desert. Journal of Desert Research, 33, 1299-1304. (in Chinese with English abstract) [杨兴华, 何清, 艾力·买买提依明, 霍文, 刘新春 (2013). 塔克拉玛干沙漠东南缘沙尘暴过 程中近地表沙尘水平通量观测研究. 中国沙漠, 33, 1299-1304.]

Yu GM, Liu Y, Yan Y, Hu YF (2011). Soil wind erosion risk assessment in the middle part of Inner Mongolia Plateau during 2000 to 2008. Scientia Geographica Sinica, 31,
1493-1499. (in Chinese with English abstract) [于国茂, 刘越, 艳燕, 胡云锋 (2011). 2000-2008年内蒙古中部地 区土壤风蚀危险度评价. 地理科学, 31, 1493-1499.]

Zhang XL, Zhou QQ, Chen WW, Wang YY, Tong DQ (2015). Observation and modeling of black soil wind-blown erosion from cropland in northeastern China. Aeolian Research, 19, 153-162.

Zhao CX, Zheng DW, He WQ (2005). Vegetation cover changes over time and its effects on resistance to wind erosion. Acta Phytoecologica Sinica, 29, 68-73. (in Chinese with English abstract) [赵彩霞, 郑大玮, 何文清 (2005). 植被覆盖度的时间变化及其防风蚀效应. 植物 生态学报, 29, 68-73.]

Zobeck TM, Stout JE (1996). The wolfforth field experiment: A wind erosion study. Soil Science, 161, 616-632.

Zou XY, Zhang CL, Cheng H, Kang LQ, Wu XX, Chang CP, Wang ZL, Zhang F, Li JF, Liu CC, Liu B, Tian JL (2014). Classification and representation of factors affecting soil wind erosion in a model. Advances in Earth Science, 29, 875-889. (in Chinese with English abstract) [邹学勇, 张 春来, 程宏, 六力强, 吴晓旭, 常春平, 王周龙, 张峰, 李继峰, 刘辰琛, 刘博, 田金鹭 (2014). 土壤风蚀模型 中的影响因子分类与表达. 地球科学进展, 29, 875889.]

责任编委: 梁存柱 责任编辑: 王 葳

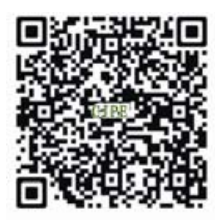

植物生态学报官网

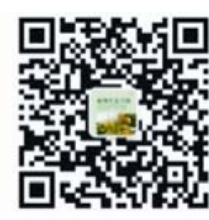

微信订阅号

期刊及学科

相关信息发布

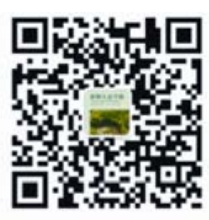

微信服务号

稿件状态查询

全文检索汶览 